\title{
Computer Control System of Crude Distillation Unit*
}

\author{
by Tahafumi Kuriyama**, Hironichi Migoshi**, Noriyoshi Ohnishi**, \\ Kazuo Amezawa***, and Katsutada Kato***
}

\begin{abstract}
Summary: I computer control system of a crude distillation unit with a design capacity of 150,000 BPSD has been developed. The crude distillation unit consists of a topping unit and a gas recovery unit. The main purpose of the system is to optimize the former unit and to control products quality of the latter unit. In either case, most of the system has been put to practical use, and its application has proved to be satisfactory. The system concept and structure are described, and the usefulness of this system has been substantiated by the results of applications.
\end{abstract}

\section{Introduction}

Recently, data processing and control with the aid of a computer have become indispensable to the petroleum refining industry in which units are increasing in capacity and complexity. It the present time, computer control finds only limited applications in a unit that is large in capacity, that produces valuable products or that is characterized by unstable operation such as a topping unit ${ }^{1 \sim 3)}$, a fluid catalytic cracking unit $^{4}$ ) and a gas recovery unit ${ }^{5}$. However, the application of the system to the simultaneous control of interconnected processes has been very limited.

Chiyoda Chemical Engineering \& Construction Co., Ltd. and Showa Yokkaichi Sekiyu Co., Ltd. have jointly developed a computer control system for a crude distillation unit with a design capacity of 150,000 BPSD, consisting of a topping unit and a gas recovery unit. This system aims to stabilize and optimize the operation of these two units using an on-line, closed-loop system, incorporating an IBM 1800 computer. The objective of the system will be achieved by the set point control (SPC) which provides information for the adjustment of analog controller settings. This paper describes the system concept and structure and some of the applications of the system.

\section{Outline of Grude Distillation Unit}

As shown in Fig. 1, the crude distillation unit

* Received October 7, 1974.

** Chiyoda Chemical Engineering \& Construction Co., Ltd. (1580 Tsurumi-cho, Tsurumi-ku, Yokohama 230)

*** Showa Yokkaichi Sekiyu (ir,, Ltd. (1 Shiohamacho, Yokkaichi 510). consists of a topping unit and a gas recovery unit. The outline of each unit is as follows:

\subsection{Topping Unit}

The crude is separated into the liquid and vapor in a preflasher. Subsequently, the liquid portion is heated to the required temperature by heat exchangers and a furnace, and is fed to a main fractionator. The vapor from the preflasher is directly fed to the main fractionator. The overhead vapor containing liquefied peroleum gas (LPG) is processed in the gas recovery unit. The side-streams such as kerosene, light gas oil and heavy gas oil withdrawn at various levels of the column are fed to the respective strippers, where the light ends are stripped. The oils may further be processed elsewhere as required. The long residue from the bottom of the column is used as a heating medium for the reboilers, and is cooled by exchanging heat with the incoming crude oil, and finally fed to a vacuum flasher.

The fractionation power between the products of the column can be manipulated with the three side refluxes-top, middle and lower-and the three side strippers-a reboiled stripper for the kerosene, and two steam strippers for the gas oils.

\subsection{Gas Recovery Unit}

The gas recovery unit is a distillation train comprising a stabilizer, a deethanizer and a depropanizer. The overhead product of the topping unit is separated into naphtha and LPG fraction in the stabilizer. The LPG fraction is split into propane and butane in the depropanizer after removing the ethane and lighter materials as off-gas in the deethanizer. The heat necessary for the reboiler of each column is supplied by the long residue of the topping unit. 


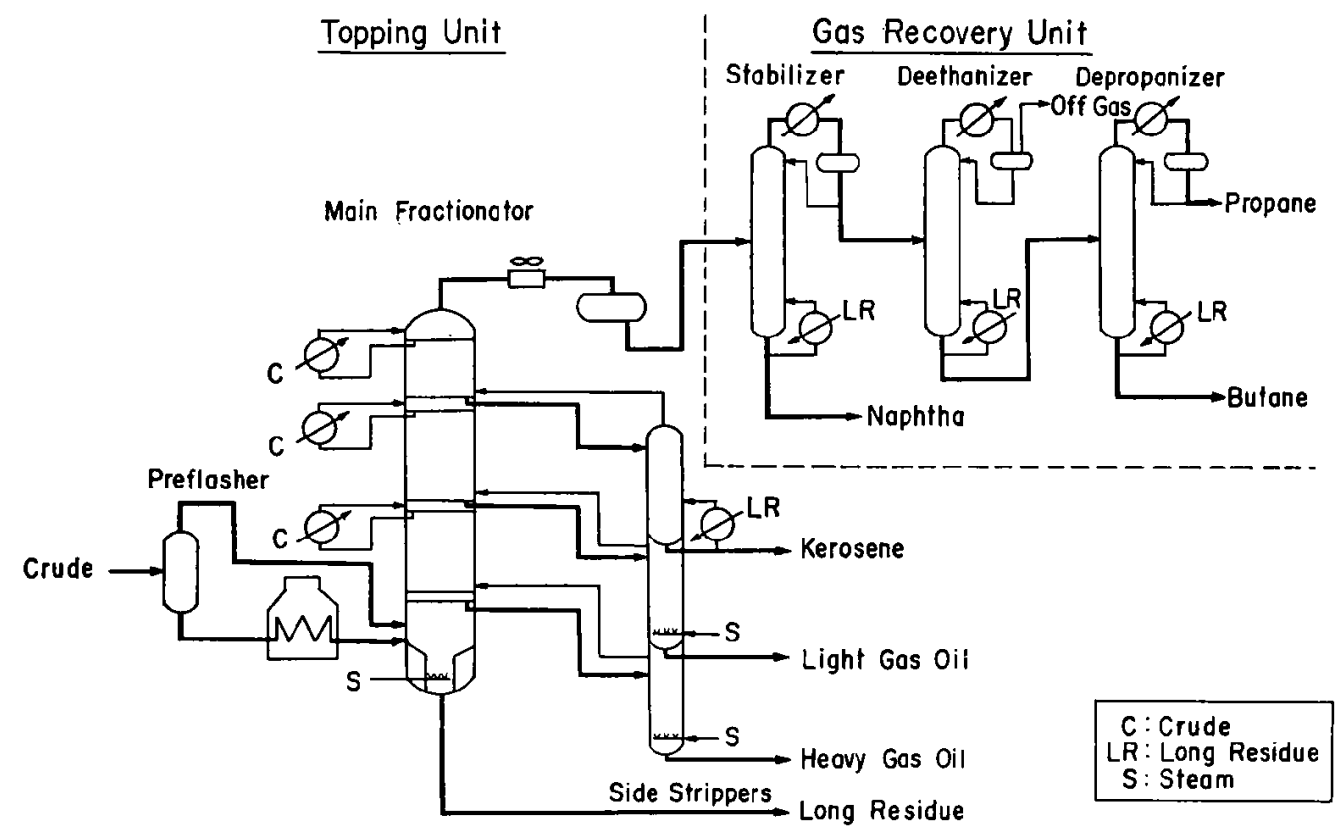

Fig. 1 Flow Diagram of Crude Distillation Unit

\section{System Concept}

Under the existing serious energy shortages and pollution problems, the plant operators will be required not only to increase profits but also to operate the plant so as to meet the changes of the operational environments. In addition, a large-scale and integrated plant should be operated with the prime consideration to manpower saving. Therefore, the objective of computer control is to improve the operability of a plant, to establish operating safety and to generate profits.

In order to attain these objectives, the following factors should be considered:

- Improvements on adaptability to operation changes

- Stabilization of operation

- Increase in yields of products of greater marketability

- Savings in utilities

- Saving in manpower

The importance of these items varies depending on the conditions or the characteristics of a plant.

Since the change in the operating condition is frequently required in a large capacity topping unit, even a small deviation from the optimal operating condition will result in large economical losses. Therefore, the realization of the optimal operating condition is more important than the development of dynamic control for such a topping unit in view of its inherent stability characteristics. For the optimization of the topping unit, the following two modes are taken into account:

(1) To maximize the kerosene yield. (Kerosene Maximum Mode) This is a particular requirement for this topping unit.

(2) To minimize the amount of utilities consumption in the case where the yield of each product is specified. (Utility Minimum Mode)

On the other hand, the gas recovery unit, a downstream unit of the topping unit, is subjected to the changes in the operating conditions of the topping unit. In addition, since the three columns are connected in series, the operation of each column is greatly affected by that of the preceding column. Therefore, the gas recovery unit cannot be operated so stably as the topping unit. Usually, the operator concerns with the change in the feed condition by raising the product quality more than that required for the on-specification product. Therefore, the purpose of computer control for the gas recovery unit is to increase the amount of on-specification products by reducing the marginal product purity and to stabilize the plant operation by performing dynamic control against process variations. If the stabilization of operation is achieved, in the gas recovery unit where process variations are great or quickly occurring, a substantial reduction in manpower will be realized. 
In order to attain the aforesaid objectives of computer control for the crude distillation unit, a coitrol system has been developed on the basis of the following concept:

\subsection{Topping Unit}

\section{(1) Distillation Model}

Optimization will necessitate the distillation models to predict the plant performance. The basic structure of these models is to be determined on the results of simulation by using an IBM 370 computer.

\section{(2) Adaptation}

Since the distillation models will not always predict accurately the behavior of a plant, it is necessary to modify the parameters of the models on the basis of current plant data. Therefore, an on-line adaptation function is incorporated in the system.

\section{(3) Control}

It is not necessary to take into account the process dynamics owing to the inherent stable operation of the topping unit. Therefore, the control should be performed on the basis of the feedback control of the proportional and integral (PI) mode.

\section{(4) Distillation Analyzer}

Most product properties are measured by online distillation analyzers. The validity of the values measured is monitored by checking the high-low limits and trend limits to prevent receiving the false information.

\subsection{Gas Recovery Unit}

(1) Distillation Model

In general, if changes in the operating condition are large or quickly occurring, the difference between the predicted values from the models and the actual values will be large. Therefore, the distillation models are not to be employed in the control system of the gas recovery unit at the sacrifice of the possible improvement on the control performance.

(2) Control

The control of product qualities is mainly performed by feedback control using the on-line gas chromatographs. Besides the feedback control, the feedforward control is added to the system to effectively compensate for the changes in the feed rate or composition.

(3) Manipulated Variable

The results of the simulation and the plant data indicate that in the distillation column, the product qualities are more sensitive to the changes in material balance than to those in separation power. Therefore, the distillate rate is to be selected as a primary manipulated variable.

(4) Control Algorithm

The use of a gas chromatograph for feedback control causes deadtime in the feedback loop. In addition to the feedback control of PI mode, Smith's control ${ }^{6}$ ) and bang-bang control ${ }^{7}$ ) are provided to solve the problem.

\section{System Structure}

In general, the multi-level approach proposed by Lefkowitz ${ }^{8)}$ is useful as practical means to design and implement an optimal control system for a complicated system. In this theory, a control system is ideally composed of four layers each with an appropriate function in the control system as shown below:

- First layer Regulation function

- Second layer Optimizing function

- Third layer Adaptive function

- Fourth layer Self-organizing function

Figure 2 shows the four-layer control configuration.

The system shown in Fig. 3 has been developed to realize the system concept as previously described. According to this principle, the system is decomposed into four parts according to their functions. Figure 3 outlines the system structure of the topping unit. The system structure of the gas recovery unit is the same as that of the topping unit, except the optimizing and adapting functions. The heat duty control and the quality control, optimization and adaptation correspond to the first, second, and third layers, respectively. The fourth layer corresponds to a retirement function though not shown in Fig. 3. Furthermore, the process monitoring function is provided to support the function of each layer. The following outlines the monitoring, the heat duty control, the quality control, optimization and adaptation:

\subsection{Monitoring}

The status of each equipment is identified with the following calculations based on process variables such as flow rate, temperature, pressure, composition and so on.

(1) Data Processing

- Smoothing of scanned data

- Checking of high-low limits and trend limits

(2) Balancing

Bulletin of The Japan Petroleum Institute 


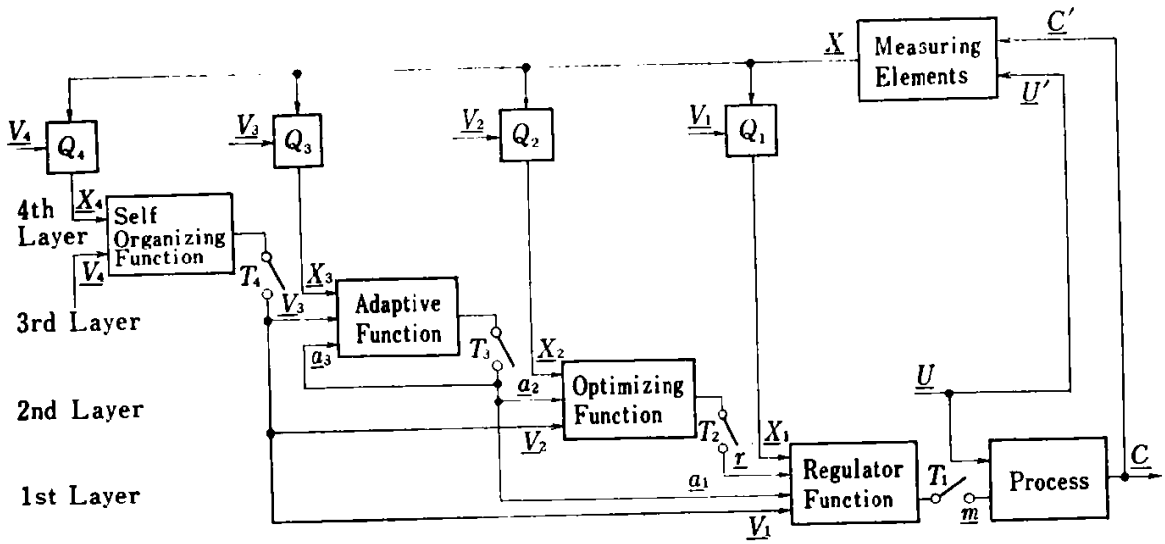

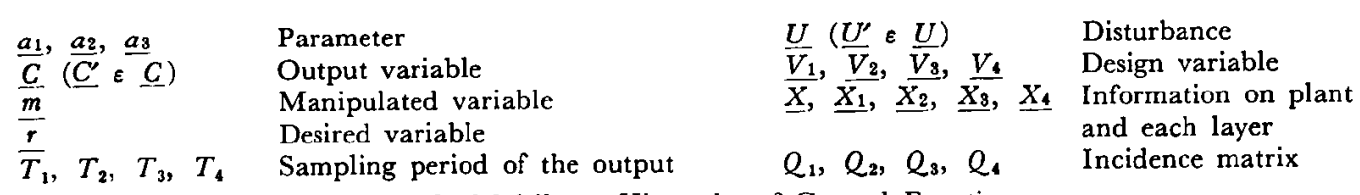

Fig. 2 Multilayer Hierarchy of Control Functions

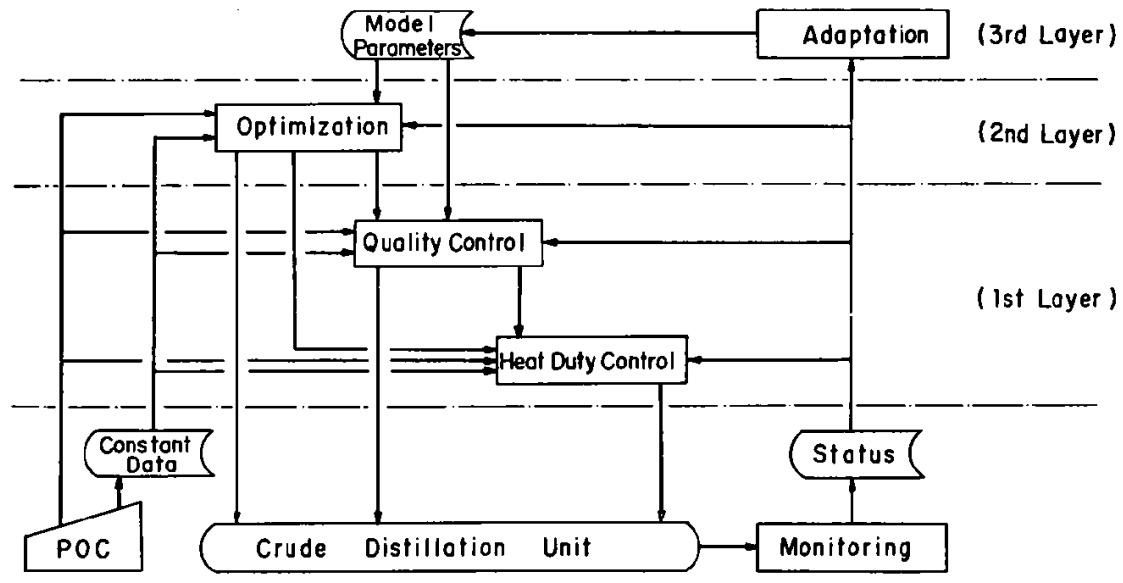

Fig. 3 System Structure for Topping Unit

- Calculation of material and heat balances concerning various kinds of envelopes.

- Calculation of vapor and liquid loads

- Calculation of reboiler and condenser heat duties

- Calculation of internal reflux ratios

- Calculation of various kinds of yields

- Estimation of unmeasurable physical properties

(3) Checking of Tray Load

- Checking as to whether the tray load is within the proper zone

- Calculation of the permissible variations of tray loads

The calculations as listed above are performed, and the results are stored in data files at a certain interval. The stored data are employed at each layer. Also, the data are displayed on a color cathode ray tube (CRT) display device through the process operator's console (POC). It is possible to provide the operators instantaneously with current information on the operating conditions, and also help them get well aquainted with the process through the CRT display. The displayed items are classified as follows:

(1) Operation Status

- Flow chart of each unit, with present values of main variables

- Present values and averaged values of process variables

- Tray load status shown graphically

- Trend of process variables

- High-speed scanned data

(2) Control Status 
- On-control loops, their set points, present values and control parameters

Figure 4 and Fig. 5 show examples of operation status on the graphic display.

\subsection{Heat Duty Control}

The outline of the heat duty control as the first layer is shown in Fig. 6 and Fig. 7. The heat duties of side refluxes and reboilers are controlled to maintain the desired value set by the POC, the quality controller or the optimizer.

For the topping unit, in addition to the above heat duty control, various kinds of monitoring

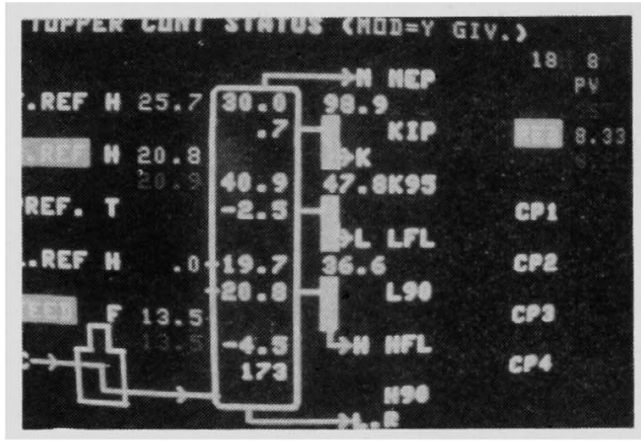

Fig. 4 Operating Status of Topping Unit for the top reflux are carried out to operate within a feasible operating region. Also, in order to compensate for changes in crude charge rate caused by variations in temperature of the preflasher, the control for crude charge rate is performed. Variations except for those in crude quality could be overcome by performing the above-mentioned controls, and the basic stable operation would be achieved.

On the other hand, for the gas recovery unit,

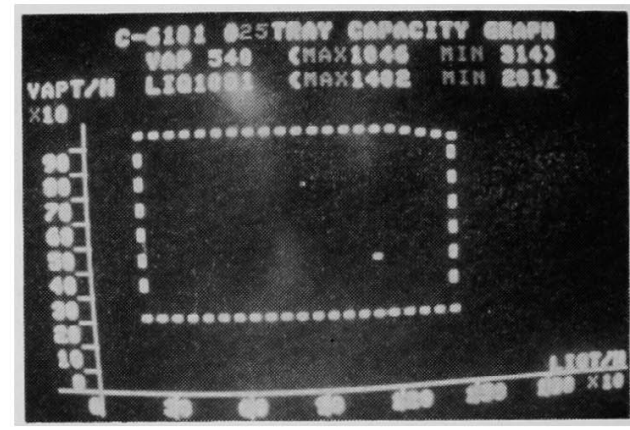

The inside of the envelope is the proper zone. The point in the envelope donotes the current operating point.

Fig. 5 Load Status of Main Fractionator $=25$ Tray

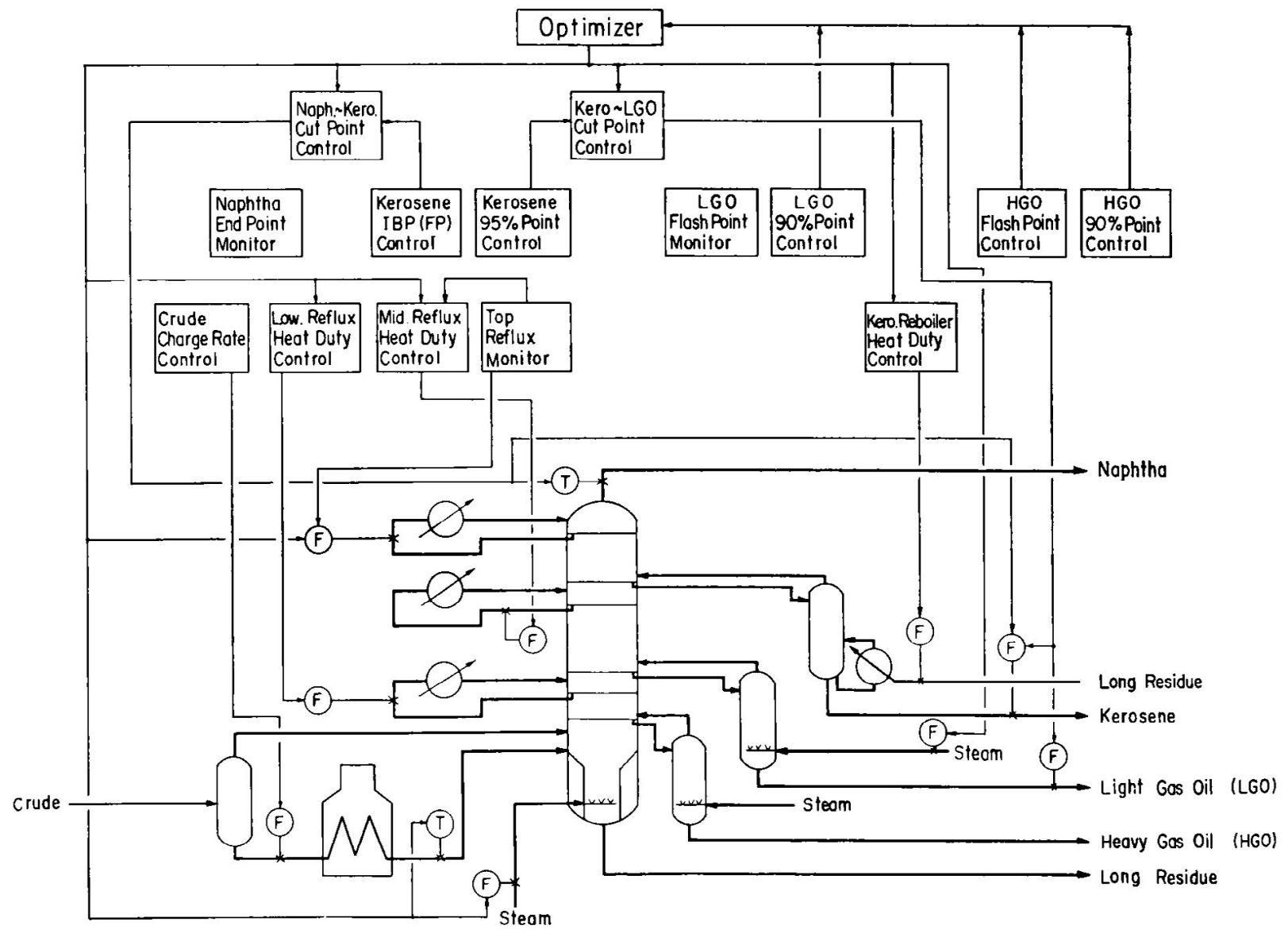

Fig. 6 Control Scheme of Topping Unit for Kerosene Maximum Mode 


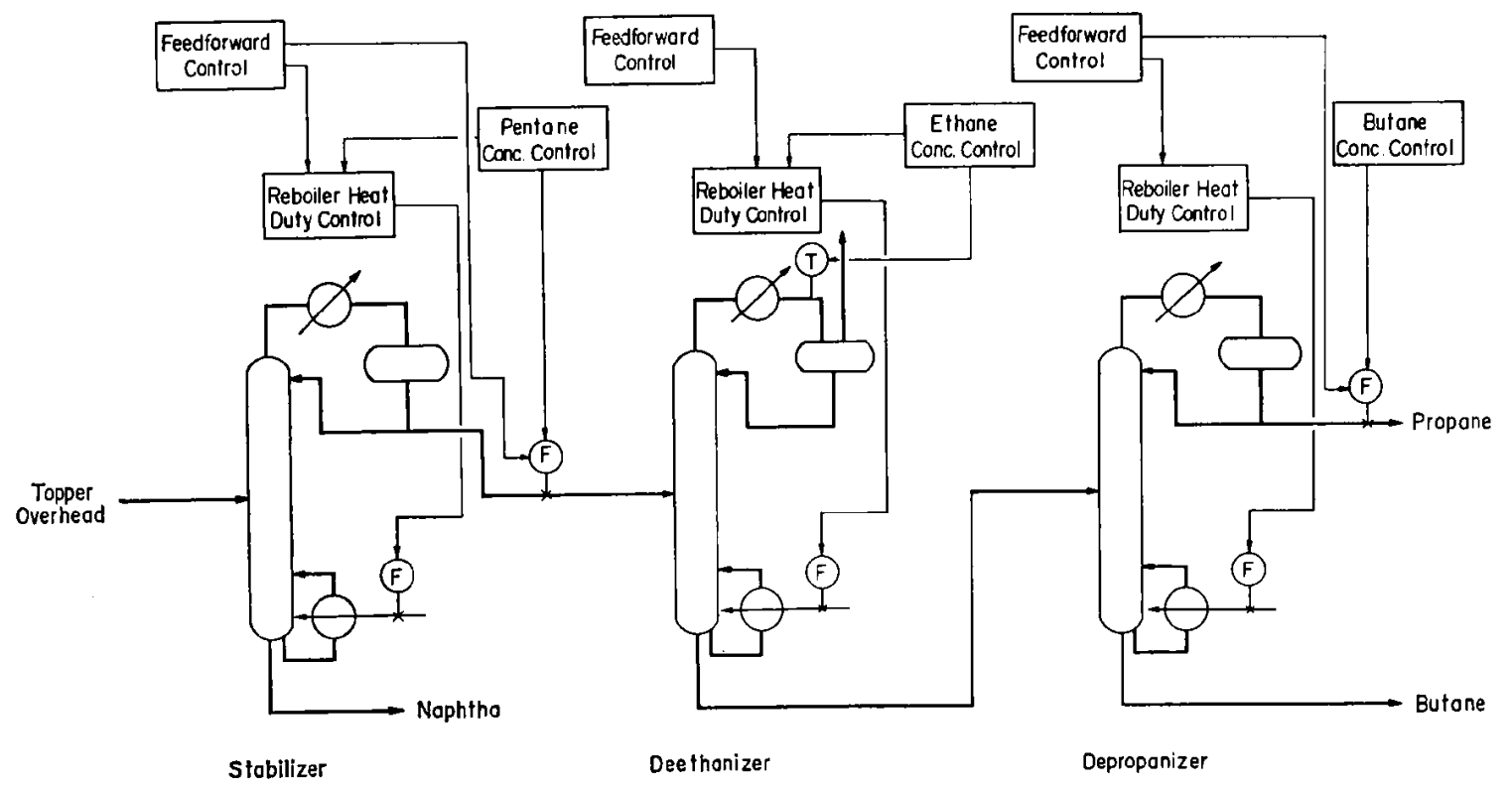

Fig. 7 Control Scheme of Gas Recovery Unit

the heat duty controller would compensate for temperature variations of the long residue which supplies heat to the reboilers.

\subsection{Quality Control}

The quality controllers as the first layer shown in Fig. 6 and Fig. 7 control various kinds of product qualities to maintain the products on specification. The means of these controllers are described below:

\subsubsection{Topping Unit}

ASTM end point of naphtha, initial boiling point* or flash point* and 95\% point of kerosene, and flash point* and $90 \%$ point of gas oils are controlled to meet each specification. (*denotes estimated value.)

(1) In the case of Kerosene Maximum Mode, the cut points between the kerosene and the adjacent fractions are adjusted to maintain the kerosene on specification. In this case, the end point of naphtha and the flash point of light gas oil would always satisfy the specification and therefore, these values are only monitored.

(2) In the case of Utility Minimum Mode, internal reflux ratios, or stripping powers obtained from a reboiler heat duty and flow rates of stripping steam are manipulated to keep the products on specification.

(3) In both modes, the control action for the ASTM 90\% point of the light gas oil, and for the flash point and $90 \%$ point of heavy gas oil would not satisfy quality specifications of the other products. In such a situation, the optimizer should be operated to control these points. The optimizer would calculate the desired manipulated values based on the entire operating condition of the topping unit so as to meet all product specifications.

\subsubsection{Gas Recovery Unit}

The objective of the quality controller is to control the pentane concentration in the distillate of the stabilizer, the ethane concentration in the bottoms of the deethanizer and the butane concentration in the distillate of the depropanizer. These controls are performed by the following means.

(I) In order to meet the specifications of the components which are given through the POG, the distillate rate, the reboiler heat duty and the distillate rate are manipulated in the stabilizer, the deethanizer and the depropanizer, respectively, with the aid of gas chromatographs.

(2) In general, if large variations in feed rate or composition occur, the feedback controllers themselves cannot sufficiently compensate for the effects of those variations. In this situation, the feedforward controller will be effective, to cope with these variations. In this controller, the distillate rate and the reboiler heat duty are employed as manipulated variables.

(3) In addition, for the stabilizer and the deethanizer, the reboiler heat duty or the condenser outlet temperature is manipulated to 
shift the operating condition of the column into a stable region and keep it in the stable region.

\subsection{Optimization}

The optimal operation of the topping unit can be achieved by the optimizer on the second layer.

This optimizer determines the basic manipulated values in correspondence with the following operating modes mainly to cope with changes in crude quality:

- Kerosene Maximum Mode-Product draw-off rates

- Utility Minimum Mode-Internal reflux ratios and stripping powers

The optimizer consists of a search routine for an optimal point and a simulation routine. The simulation routine simulates the entire behavior of the topping unit including the main fractionator, the side strippers, the preflasher, the furnace and the heat exchangers, and checks against various kinds of limitation, and consequently determines the feasible operating conditions and calculates the value of the objective function. On the other hand, the search routine finds a set of the manipulated variables, giving the maximum or the minimum value of the objective function by a hill climbing method. The method is the Box method") partially modified for this work, which was chosen because of the easiness of programming and the reliability in searching an optimal point.

In addition to the periodical running of the optimizer, if various kinds of requirement to the duty controllers or the quality controllers could not be satisfied in each controller, its running can be especially requested.

\subsection{Adaptation}

There exists a function adapting the parameters of the distillation models for the topping unit as the third layer. The distillation models are the relationships between ASTM distillation temperatures and the manipulated variables-cut points, reflux ratios and stripping powers. These models are constructed as linear combinations of a first-order polynomial function and a first-order fractional function. The models were derived from the simulation studies and therefore, they have to be checked and corrected based on the actual performance of the plant.

The deviations between the model-estimated values and the measured values are stored in the file. The bias terms of the models are updated on the basis of the moving-averaged values of the

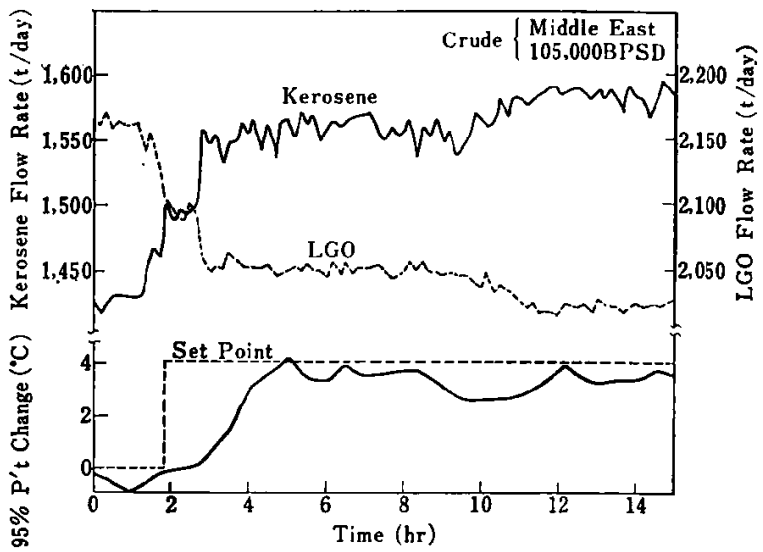

Fig. 8 Performance of Kerosene 95\% Point Control

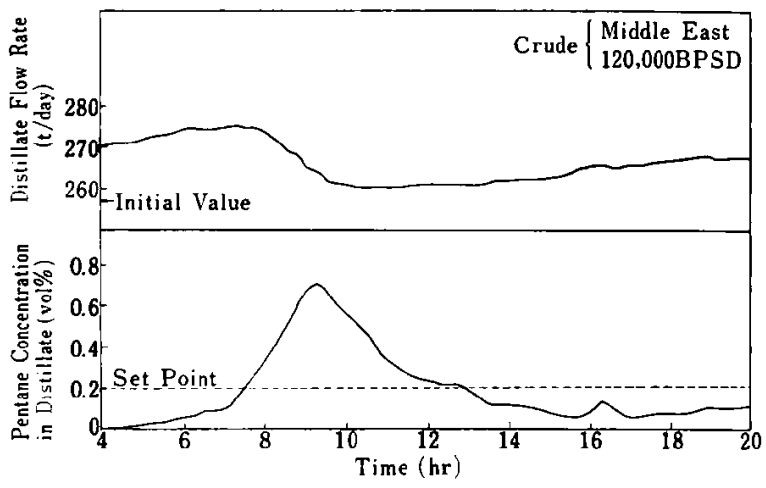

Fig. 9 Performance of Quality Control for Stabilizer

above stored deviations. The updating of the parameters is performed each time that the product qualities are measured. If the extent of parameter modification exceeds the given limits, an alarm message is typed, and the data are cancelled.

\section{Results of Application}

The testing of the system has been carried out step by step from the monitoring to the optimization. Some results of the application are described below:

Figure 8 shows a result of $95 \%$ point control of kerosene for Kerosene Maximum Mode. In this operation, an increase in the kerosene yield was aimed by raising a set point of $95 \%$ point by $4^{\circ} \mathrm{C}$. The $95 \%$ point settled in a short time, and the draw-off rate of the kerosene increased as expected. About $1 \%$ increase in the kerosene yield on crude has been achieved.

Figure 9 shows a result of the feedback control for the pentane concentration in the stabilizer distillate. This was the case where the set point of the pentane concentration was changed from 0 to $0.2 \mathrm{vol} \%$. It proves very difficult from 


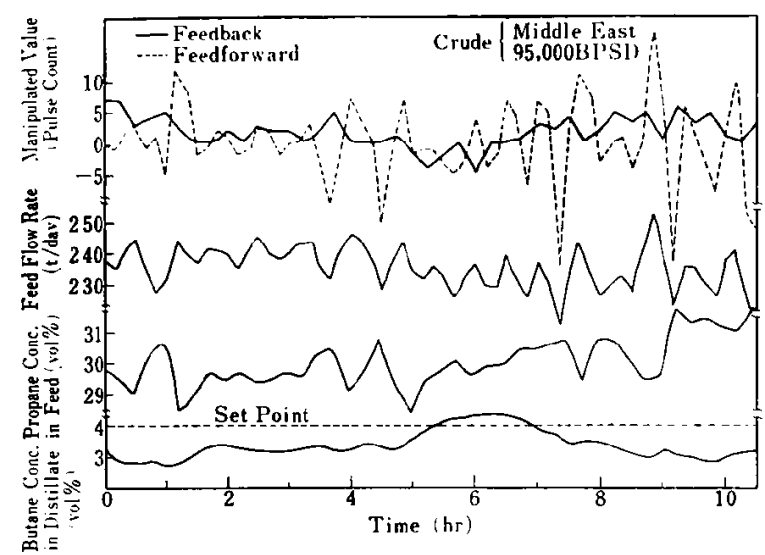

Fig. 10 Performance of Quality Control for Depropanizer

our experience that the increase in the recovery of butane is achieved by adding pentane of no more than $0.2 \mathrm{vol} \%$ to the distillate of the stabilizer. Therefore, it was a great success that the pentane concentration could be increased up to $0.2 \mathrm{vol} \%$ and maintained within a range of 0 to $0.2 \mathrm{vol} \%$ with the aid of a computer.

Figure 10 shows a result of the combined feedforward-feedback control for the butane concentration in the depropanizer distillate. Since this column is subjected to operating condition of the preceding columns, the combined control is very useful in overcoming possible disturbances. The distillate rate was manipulated to compensate for the changes in feed condition shown in Fig. 10, and consequently the butane concentration in the distillate was maintained close to the set point. The implementation of the system resulted in about $2 \%$ increase in the recovery of propane and about $4 \%$ in that of butane over the recovery rates in the conventional operation. The computer expenses can be covered by the profits from the gas recovery unit.

\section{Discussion}

(1) The adequate communication of the operators with the process through the CRT display has played an important role in developing the system.

(2) In general, a flexible and simplified model is practically useful to computer control. This was proved in the application to this topping unit. The models derived from the simulation studies were found covering the range from the half load operation to the full.

(3) Though the system has been developed for Kerosene Maximum Mode as one mode of optimization, the maximization of the yield of the other products could be similarly performed under certain process conditions.

(4) So far, it has not been found that there exists a remarkable difference in control performance among PI control, Smith's control and bangbang control for the gas recovery unit. However, since sufficient data for the minute comparisons with these algorithms have not been obtained, further study on this problem will be conducted.

(5) The combined feedforward-feedback control is provided for each column of the gas recovery unit. The feedforward control was very effective, particularly, to the depropanizer.

(6) The development of the system heavily depends on the stream analyzers. The suitable selection and adequate maintenance of these analyzers served as a key element to the success in developing the system.

(7) Though some points to be modified have been found in the testing of the system, the basic approach to the development of the system has proved to be suitable to the purpose. However, further improvements on this system are to be made. The results will be reported on another occasion.

\section{Conclusions}

The computer control system of the crude distillation unit has been developed and has effectively been utilized.

The optimal operation of the topping unit can be realized by implementing this system. For the gas recovery unit, the tight control has been achieved despite frequent changes in operating conditions and the assurance of the product qualities and manpower saving have been achieved.

Also, the installation of an on-line control computer in the crude distillation unit has economically been justified.

\section{Acknowledgement}

The authors express sincere appreciation to Messrs. Masami Yamamoto, Shizuaki Ozawa and Jotaro Ito of Chiyoda Chemical Engineering \& Construction Co., Ltd., and also to Messrs. Shigeru Imamura, Haruo Suzuki and other personnel engaging in the instrumentation and the plant operation in Showa Yokkaichi Sekiyu Co., Ltd. for their untiring support in developing this system.

They also wish to acknowledge Chiyoda Chemical Engineering \& Construction Co., Ltd. and 
Showa Yokkaichi Sekiyu Co., Ltd. for permission to publish this paper.

\section{References}

1) Crowther, R. H., Pttrak, J. E., Ply, E. N., Chem. Eng. Progr., 57, (6), 39 (1961).

2) Adams, G. F., Oil Gas J., 60, (21), 114 (1962).

3) Bridgewater, K., Flint, F. C., Hambury, J. N., Sagues, J. I., 3rd IFAG Congress, Paper 43C, London
(1966).

4) Snondgrass, J. M., Smith, R. A., Gossett, E. C., Plys, A. G., Hydrocarbon Processing, 47, (5), 108 (1968).

5) Konishi, M., Automation, 10, (13), 69 (1965).

6) Smith, O. J. M., ISA Journal, 6, (2), 28 (1959).

7) Zoss, L. M., Mortimer, K., Vetter, K. H., Instrumentation Technology, April 59 (1970).

8) Lefkowitz, I., Trans. ASME, 88, (6), 1 (1966).

9) Box, M. J., Computer Journal, 8, (1), 42 (1965). 